\title{
Imaging heterochromatin in human embryonic stem cells with light-sheet Bayesian microscopy
}

\author{
Ying S Hu', Quan Zhu', James Fitzpatrick', Inder M Verma², Hu Cang ${ }^{1 *}$ \\ From Epigenetics \& Chromatin: Interactions and processes \\ Boston, MA, USA. 11-13 March 2013
}

We present a novel super-resolution microscope for the study of chromatin architecture. In eukaryotic cells, chromatin is organized into two distinct domains: lightly packed, and actively transcribed euchromatin, and highly condensed, and transcriptionally silent heterochromatin. While being transcriptionally repressive, heterochromatin is known to serve as binding sites for regulatory proteins, and therefore, plays an important role in the epigenetic regulation of gene expression [1]. However, despite its importance, the architecture of heterochromatin remains elusive, due to the lacking of powerful tools. Single-molecule super-resolution microscopy [2,3], invented by Betzig [4], Hess [5], and Zhuang [6], improves the spatial resolution of light microscopy by over an order of magnitude to $\sim 20-30 \mathrm{~nm}$, and therefore offers a new approach to examine the 3D architecture of chromatin. However, its fundamental requirement of high signal-to-noise ratio (SNR) of fluorescence signal to detect single fluorophore has limited its applications mainly to the study of the cytoskeleton and membrane proteins that reside near the surface of a cell [7-9]. To image beyond the surface of a cell where chromatin resides, we develop a Light-Sheet Bayesian Superresolution Microscope (LSB-SRM). This microscope is a marriage between two recently developed techniques: light-sheet single molecule super-resolution microscopy and Bayesian super-resolution image reconstruction algorithm. The light-sheet condenser selective illuminates the nucleus of a cell, suppresses the fluorescence background from fluorophore in unwanted layer of the cell, and therefore increases the signal-to-noise ratio of the single-molecule detection. The Bayesian algorithm can efficiently resolve single molecules in images with noisy background. A combination of the two allows us to directly visualize heterochromatic structures in the nucleus of human

${ }^{1}$ Waitt Advanced Biophotonics Center, Salk Institute for Biological Studies,

La Jolla, CA 92037, USA

Full list of author information is available at the end of the article embryonic stem cells at super-resolution. Our study shows that the heterochromatin organize into nanometer scale domain structures in H1, human embryonic stem cells, which is consistent with the topological domain theory derived from recent sequencing experiments $[10,11]$.

\section{Author details}

${ }^{1}$ Waitt Advanced Biophotonics Center, Salk Institute for Biological Studies, La Jolla, CA 92037, USA. ${ }^{2}$ Laboratory of Genetics, Salk Institute for Biological Studies, La Jolla, CA 92037, USA.

Published: 8 April 2013

\section{References}

1. Maison C, Almouzni G: HP1 and the dynamics of heterochromatin maintenance. Nat Rev Mol Cell Biol 2004, 5:296-304.

2. Patterson G, Davidson M, Manley S, Lippincott-Schwartz J: Superresolution imaging using single-molecule localization. Annual Review of Physical Chemistry 2010, 61:345-367.

3. Moerner WE: New directions in single-molecule imaging and analysis. Proceedings of the National Academy of Sciences 2007, 104:12596-12602.

4. Betzig $E$, et al: Imaging Intracellular Fluorescent Proteins at Nanometer Resolution. Science 2006, 313:1642-1645.

5. Hess ST, Girirajan TPK, Mason MD: Ultra-High Resolution Imaging by Fluorescence Photoactivation Localization Microscopy. Biophysical Journal 2006, 91:4258-4272.

6. Rust MJ, Bates M, Zhuang XW: Sub-diffraction-limit imaging by stochastic optical reconstruction microscopy (STORM). Nature Methods 2006, 3:793-795.

7. Huang B, Wang W, Bates M, Zhuang X: Three-Dimensional SuperResolution Imaging by Stochastic Optical Reconstruction Microscopy. Science 2008, 319:810-813.

8. Kanchanawong $P$, et al: Nanoscale architecture of integrin-based cell adhesions. Nature 2010, 468:580-584.

9. Xu K, Babcock HP, Zhuang X: Dual-objective STORM reveals threedimensional filament organization in the actin cytoskeleton. Nat Methods 2012, 9:185-188.

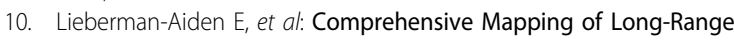
Interactions Reveals Folding Principles of the Human Genome. Science 2009, 326:289-293.

11. Dixon $J R$, et al: Topological domains in mammalian genomes identified by analysis of chromatin interactions. Nature 2012, 485:376-380.

doi:10.1186/1756-8935-6-S1-P109

Cite this article as: Hu et al:: Imaging heterochromatin in human embryonic stem cells with light-sheet Bayesian microscopy. Epigentics \& Chromatin 2013 6(Suppl 1):P109. 